In summary microglial AICD is accompanied by upregulated expression of pro-apoptotic proteins and is mediated mainly via NO-promoted Bak/Bax activation and the mitochondrial gateway.

Acknowledgements. We thank Dr. Thomas Möller for helpful advices. This work was supported by the Israel Science Foundation and the Adams SuperCenter for Brain Research.

\author{
$L_{\text {Mayo }}{ }^{\text {and } R}$ Stein $^{\star, 1}$ \\ 1 Department of Neurobiochemistry, George S. Wise Faculty of Life Sciences, \\ Tel Aviv University, 69978, Ramat Aviv, Israel
}

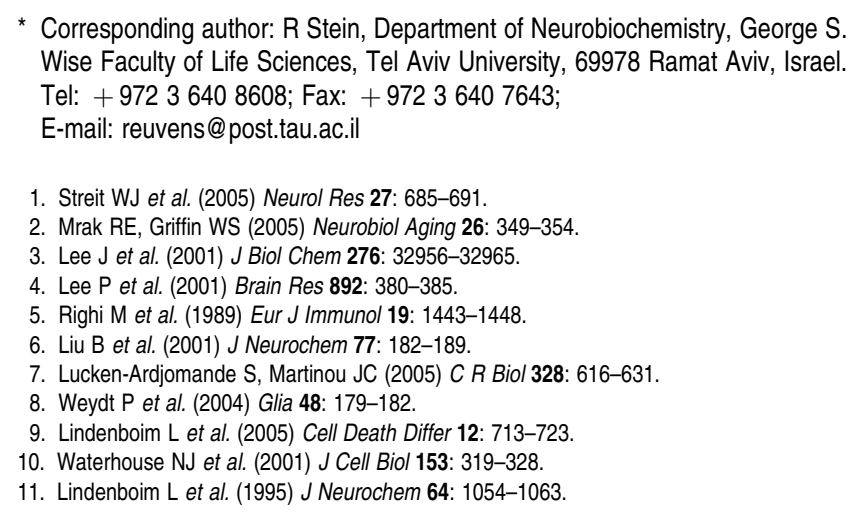

* Corresponding author: R Stein, Department of Neurobiochemistry, George S. Tel: + 97236408608 ; Fax: + 97236407643

E-mail: reuvens@post.tau.ac.il

1. Streit WJ et al. (2005) Neurol Res 27: 685-691.

2. Mrak RE, Griffin WS (2005) Neurobiol Aging 26: 349-354.

3. Lee J et al. (2001) J Biol Chem 276: 32956-32965.

8. Weydt P et al. (2004) Glia 48: 179-182.

11. Lindenboim L et al. (1995) J Neurochem 64: 1054-1063.

\title{
Compensatory mechanisms regulate the Bcl-2 rheostat and lymphocyte survival in the absence of $\mathrm{AKT} 1 / \mathrm{PKB} \alpha$
}

Cell Death and Differentiation (2007) 14, 186-189. doi:10.1038/sj.cdd.4402000; published online 16 June 2006

\begin{abstract}
Dear Editor,
The serine-threonine protein kinase AKT (PKB) plays a central role in controlling essential cellular processes, including proliferation, glucose metabolism, angiogenesis and survival (for a review see Datta et al. ${ }^{2}$ ). Three major isoforms, $\mathrm{AKT} 1 / \mathrm{PKB} \alpha, \mathrm{AKT} 2 / \mathrm{PKB} \beta$ and $\mathrm{AKT} 3 / \mathrm{PKB} \gamma$, encoded by independent genes exist in mammals. All three isoforms are ubiquitously expressed although the levels of expression vary among tissues and AKT1 appears to be the predominant isoform. ${ }^{2}$ Studies in knockout mice lacking individual genes implicate specific, only partially overlapping functions for the different AKT isoforms. Mice lacking akt1 exhibit impaired growth with a reduction in body weight of $30 \%$ and a proportional decrease in the sizes of all major organs, identifying AKT1 as a key regulator of organismal growth. ${ }^{3,4}$ Thymocytes and mouse embryonic fibroblasts (MEF) derived from these animals were reported to display an increased susceptibility to spontaneous and stress-induced apoptosis. ${ }^{4}$ By contrast, akt2-deficient mice exhibit insulin resistance and a diabetic phenotype but are only mildly growth retarded. ${ }^{5}$ AKT2, therefore, is the relevant isoform controlling insulin regulated glucose metabolism. So far, apoptosis defects have not been reported in cells derived from mice lacking either akt2 or akt3. ${ }^{5-7} A k t 1$ and akt1/2 double deficient MEF, transformed by a dominant-negative version of p53 succumbed to cell death with identical kinetics when treated with the hexokinase inhibitor clotrimazole, indicating that
\end{abstract}

loss of akt2 does not further increase the apoptosis susceptibility of akt $1^{-1-}$ cells. $^{8}$

AKT-mediated signals have been reported to suppress apoptosis by modulating the expression and/or function of a series of pro- and antiapoptotic genes, many of which belonging to the $\mathrm{Bcl}-2$ family. ${ }^{9}$ In line with these observations, loss of $b c l-2, m c l-1$ or $b c l-X_{L}$ in mice causes depletion of lymphocytes, myeloid cells and erythroid progenitors, respectively, due to increased susceptibility of these cells to trophic factor deprivation. ${ }^{10}$ As loss of akt1 was reported to render thymocytes and primary MEF more susceptible to spontaneous as well as stress-induced cell death triggered by serum deprivation, glucocorticoids and $\gamma$-irradiation, ${ }^{4}$ we reasoned that the molecular basis for this phenomenon may be deregulated expression and/or function of certain pro- and antiapoptotic Bcl-2 family members, either repressed (e.g. Bad, Bim, or PUMA ${ }^{1,11,12}$ ) or induced (e.g. Bcl- $\mathrm{X}_{\mathrm{L}}$ or Mcl$1^{13,14}$ ) by AKT.

To explore this possibility, we analysed mRNA expression levels of pro-and antiapoptotic Bcl-2 family members in wt thymocytes or those lacking akt1. By applying quantitative real-time PCR analysis, we observed that the absence of akt1 does not impair the basal mRNA transcription of any known Bcl-2 family member in primary thymocytes. Neither an upregulation of proposed AKT-downstream targets of the proapoptotic Bcl-2 family members such as bim or puma nor a 
decrease in the transcription levels of members of the prosurvival subfamily such as $m c l-1$ or $b c l-X_{L}$ was observed in the absence of akt1 (Supplementary Figure 1).

To investigate the possibility that loss of AKT1 may affect protein stability of certain Bcl-2 family members, Western blot analysis was performed on lysates form freshly isolated wt and akt $1^{-1-}$ thymocytes. As shown in Figure 1a, Western blot analysis for AKT1 confirmed the loss of the protein in akt1-homozygous mutant mice. Expression levels of homologues AKT2 and AKT3, the latter predominantly expressed in the CNS but also weakly in lymphatic organs, were comparable between genotypes (Figure 1a). Interestingly, total AKT activity, measured by analysing the phosphorylation levels of Ser473 for AKT1, Ser474 for AKT2 and Ser472 for AKT3, all recognized by the same phospho-specific antibody was reduced in thymocytes from mice lacking akt1 by about $50 \%$ (Figure 1a). These findings indicate that loss of akt1 in thymocytes does not induce compensatory upregulation of other AKT isoforms nor does it lead to massive compensatory hyperactivation of AKT2 and/or AKT3. Our further analysis revealed that loss of akt1 expression and the resulting difference in total AKT activity is neither crucial for the maintenance of the basal expression levels of known Bcl-2 family members in primary thymocytes in vivo nor does it impinge on the activation status of known AKT downstream effectors such as Bad or the transcription factor Foxo3a, as monitored by the use of activation = phosphorylation-status-specific antibodies (Figure 1a). Thus, a modulation of the apoptotic threshold, caused by the deregulated expression of members of the Bcl-2 rheostat, may not account for the described hypersensitivity in thymocytes derived from akt1 null mice. Consistent with our findings, but in contrast to published data, ${ }^{4}$ we could not confirm an increase in spontaneous apoptosis in freshly isolated thymocytes derived from $a k t 1^{-1-}$ mice, as measured by AnnexinV/propidium iodide (PI) staining and flow cytometric analysis straight after organ harvest (Figure 1b, upper left panel). We also failed to detect an increased number of apoptotic akt1 $1^{-1-}$ cells in thymic sections in situ using TUNEL staining, when compared with sections from wt controls (Supplementary Figure 2).

These observations prompted us to re-evaluate the apoptotic response of wt and akt1-l- thymocytes in response to cytokine withdrawal (i.e. spontaneous cell death in culture), DNA-damage induced by $\gamma$-irradiation or the topoisomerase II inhibitor Etoposide (VP16), the glucocorticoid (GC) Dexamethasone or the microtubule-stabilising agent Taxol. Cell death was monitored by AnnexinV/PI staining and flow cytometric analysis. Exposure of thymocytes to $500 \mathrm{rad}$ of $\gamma$ irradiation or of $1 \mu \mathrm{M}$ of Dexamethasone induced apoptosis in more than $90 \%$ of all cells within the first $12 \mathrm{~h}$ regardless of the genotype (data not shown) and, therefore, lower doses were applied to reveal possible differences in cell death susceptibility. Still, thymocytes lacking akt1 died with similar kinetics in vitro than wt cells in response to all stimuli tested (Figure 1b). Taxol proved to be ineffective in linking thymocytes. In order to assess whether apoptotic responses would differ between the genotypes in vivo, wt and akt1 ${ }^{-1-}$ mice were exposed to $500 \mathrm{rad}$ of whole-body $\gamma$-irradiation. Thymi were harvested $20 \mathrm{~h}$ thereafter and apoptotic cells were detected in histological sections in situ by TUNEL-staining (Supplementary Figure 2). No significant differences were observed between genotypes. We further expanded our analysis to mature peripheral T lymphocytes and investigated the consequence of $A K T 1$-deficiency on IL-2 withdrawal-induced death of activated T-cell blasts, known to be dependent on the putative AKT-target Bim, ${ }^{10}$ as well as on activation induced cell death (AICD), induced by restimulation of the T-cell receptor (TCR), known to depend on Fas/FasL signaling. Both forms of cell death can be blocked by deregulating AKT activity in T cells. ${ }^{15,16}$ Our analysis showed neither accelerated spontaneous apoptosis of $A K T 1$-deficient T-cell blasts upon IL-2 withdrawal nor AICD, triggered by cross-linking of the TCR with different concentrations of anti-CD $\varepsilon$ antibodies. DNA-damage induced apoptosis by Etoposide occurred with similar kinetics in both genotypes (Figure 1c). We extended our studies on primary MEF derived from day E14.5 wt and $\mathrm{akt1^{-1- }}$ embryos and again failed to detect significant differences in death rates between the two genotypes in response to FCS deprivation, treatment with $\mathrm{TNF} \alpha$, the pankinase inhibitor Staurosporine, Etoposide, UV-irradiation or keeping the cells in suspension forcing them to undergo a distinct form of cell death, called anoikis (Figure 1d and Supplementary Figure 3).

Student's $t$-test analysis failed to reveal statistical significant differences between the two genotypes regardless of cell type, time or the cell death stimulus applied with one exception: exposure of MEF to $100 \mathrm{~J} / \mathrm{m}^{2}$ UV irradiation (Figure 1d; $p=0.015$ ). This leaves the possibility that loss of AKT1 may accelerate death in response to high, supraphysiogical doses of UV irradiation, but not at lower doses. Since such high doses of UV irradiation killed more than $85 \%$ of live cells within $24 \mathrm{~h}$, we doubt that this statistical difference is of physiological relevance, especially since it was not evident when MEF were exposed to 5 or $25 \mathrm{~J} / \mathrm{m}^{2}$ of UV irradiation and cell death quantified after 24 or $48 \mathrm{~h}$ (Figure 1d, and data not shown).

Our findings indicate that possible AKT1-mediated effects on the transcription of Bim (after IL-2 deprivation) or FasL (after TCR re-stimulation) are not essential for the execution of growth factor deprivation-induced apoptosis or AICD in T-cell blasts and can efficiently be compensated for by other means.

Our observations contradict previously published data where cells have been treated with some of the stimuli that we also included in our analysis but where cell death was either evaluated only semiquantitatively in situ using TUNEL staining of histological sections from thymi, by means of Trypane-blue exclusion after ex vivo treatment of thymocytes or an ELISA-based cell death assay for analysing MEF. ${ }^{4}$ All these methods are, in our point of view, less reliable than flowcytometric quantification of cell death using AnnexinV and PI. Currently, we have no plausible explanation for the observed differences but can only speculate that the genetic background of the mice analysed (129SV:C57BL/6 mice versus C57BL/6 back-crossed animals in our study) or off-target effects caused by the neomycine selection marker cassette may account for these effects. Loss of akt2 also appears to have different effects on the overall organismal growth on different genetic backgrounds since akt2-l- mice on the mixed 129SV/C57BL6 background show no such phenotype, whereas those generated on the DBA/1lacJ/C57BL/6 background show a growth retardation comparable to the one observed in akt1 ${ }^{-1-}$ mice. ${ }^{5,7}$ Growth retardation of AKT1- 
a
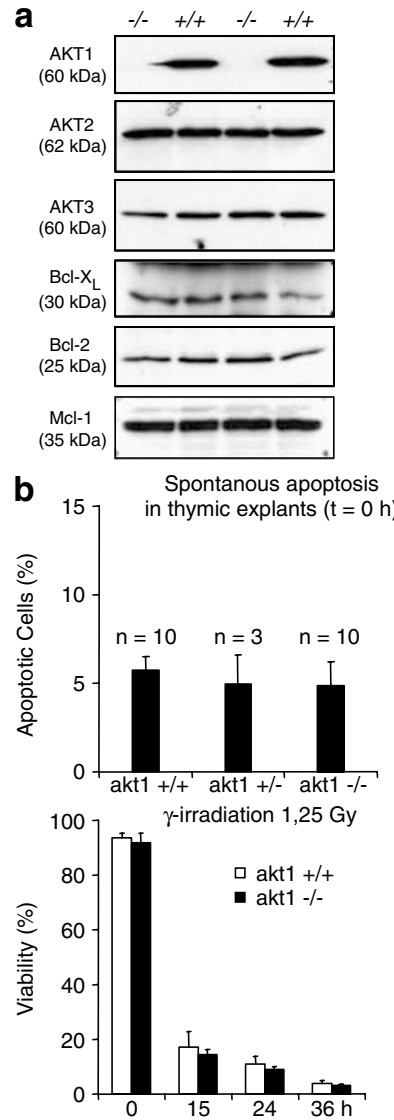

C 100

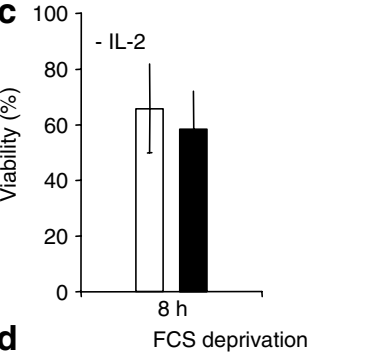

d

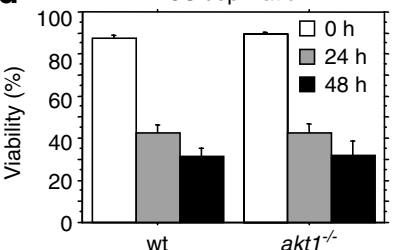

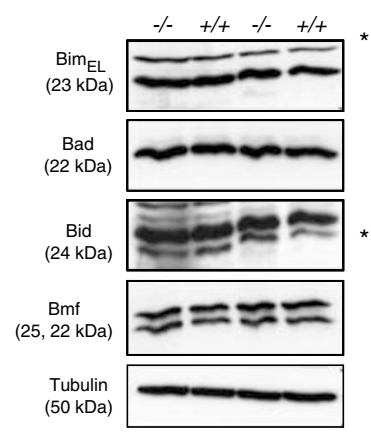
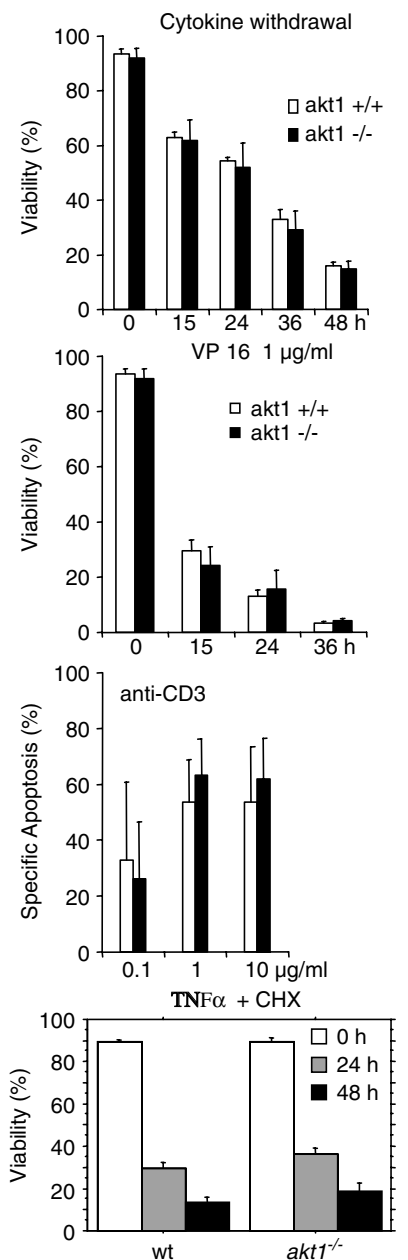

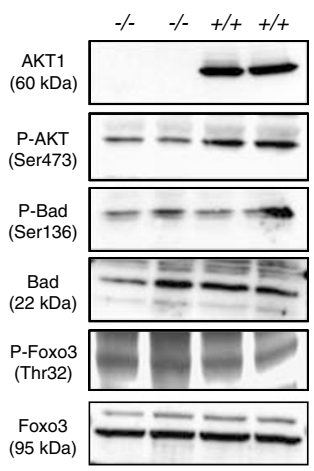

Dex $100 \mathrm{nM}$
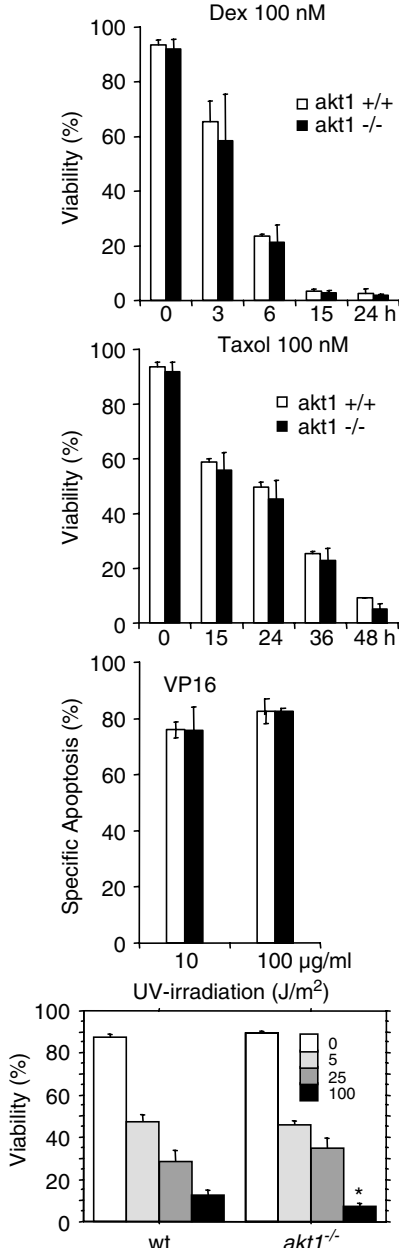

Figure 1 (a) Protein expression levels of the indicated Bcl-2 family members and the phosphorylation status of AKT-regulated downstream targets were assessed by immunoblotting analysis of total protein lysates derived from freshly isolated wt or akt thymocytes. The asterix ( ${ }^{*}$ ) indicates an unspecific band, which was also picked up with the used antibody. One out of two independent experiments yielding identical results is shown. (b) Spontaneous thymocyte apoptosis was assessed in cells derived from wt, akt $1^{+/-}$and $a k t 1^{-l-}$ thymi by FITC-AnnexinV/PI staining and flow cytometric analyis immediately after organ harvest and generation of single cell suspensions. The viability of isolated thymocytes cultured ex vivo for the indicated periods of time in the absence of cytokines (spontaneous death in culture), after exposure to $\gamma$-irradiation or in the presence of the indicated cell death-inducing agents was quantified by FITC-AnnexinV/PI staining of cell suspensions and flow cytometric analysis. Bars represent means \pm S.D. of 3-4 independent experiments using cells derived from $3 \mathrm{wt}$ and 4 akt $1^{-l-}$ animals. (c) Total splenocytes derived from akt 1 proficient or deficient mice were used to generate activated T-cell blasts using ConA $(2 \mu \mathrm{g} / \mathrm{ml}) / \mathrm{IL}-2$ stimulation $(100 \mathrm{U} / \mathrm{ml})$. After 6 days, activated T-cell blasts were washed twice, viable cells isolated by Lympholyte ${ }^{\mathrm{TM}}$ gradient centrifugation (viability $>90 \%$ ) and incubated in regular medium to assess apoptosis induced by IL-2 deprivation ( $n>6 /$ genotype). Alternatively, cells were treated with different concentrations of anti-CD3 $\varepsilon$ cross-linking antibodies (clone 2C11) to induce AICD $(n=6)$ or graded amounts of Etoposide (VP16) for $8 \mathrm{~h}(n=4)$. Apoptosis was analysed by AnnexinV/PI staining followed by flow cytometric analysis. The amount of apoptosis specifically induced by TCR ligation or drug treatment was calculated using the following equation (induced apoptosis-spontanous apoptosis)/(100-spontanous apoptosis). Bars represent means \pm S.D. (d) Early passage MEF derived from E14.5 embryos were exposed to the indicated cell death stimuli. TNF $\alpha$ was used for $10 \mathrm{ng} / \mathrm{ml}$ and CAX at $5 \mu \mathrm{g} / \mathrm{ml}$. Viability was assessed by AnnexinV/PI staining and flow cytometric analyisis after 24 and/or $48 \mathrm{~h}$. Bars represent means of 3-5 independent experiments \pm S.E. using MEF derived from four (wt) or five $\left(a k t 1^{-1-}\right)$ independent embryos that were obtained from two separate breedings 
deficient mice, however, appears to be independent of the genetic background. ${ }^{3,4}$ Genetic background differences have also been reported to drastically change the phenotype observed in mouse models lacking prominent cell death associated genes. Loss of p53 causes a very different tumour spectrum on different genetic backgrounds and embryonic lethality of female $p 53^{-1-}$ mice ranges from 30 to almost $100 \%$, due to neuronal tube-closure defects. ${ }^{17}$ Caspase- 3 or 9 deficiency cause phenotypes ranging from embryonic lethality due to massive fore-brain overgrowth to mild apoptosis defects. ${ }^{18,19}$

In conclusion, our study indicates that AKT1-mediated signals are not required to maintain the $\mathrm{Bcl}-2$ rheostat in primary mouse thymocytes. Loss of akt1 does not change the cell death susceptibility of $T$ lymphocytes and MEF to cell stresses as diverse as IL-2 or serum deprivation, TNF-RI or TCR ligation, glucocorticoids, kinase inhibition by Staurosporine and DNA-damage caused by UV- or $\gamma$-irradiation as well as topoisomerase II inhibition. This may be due to genetic background differences interfering with AKT1 regulated signaling. Finally, loss of akt1 does not cause compensatory upregulation of either AKT2 or AKT3, nor restoration of the overall AKT activity to wt levels underlining the strict separation of their true biological functions.

Acknowledgements. The work in our laboratory is supported by grants from the Austrian Science Fund FWF (SFB021, START). We thank Professor M. Birnbaum (Penn State University) for the donation of akt1 $1^{-1-}$ mice. M. Erlacher for help with the TUNEL analysis ${ }^{20}$ and all members of the SFB021 for their input in our research.
S Kiessling ${ }^{1}, C$ Lutz-Nicoladoni', A Olsson ${ }^{1}$, N Harald ${ }^{1}$, G Baier ${ }^{2}$ and $A$ Villunger ${ }^{\star, 1}$

${ }^{1}$ Division of Experimental Pathophysiology \& Immunology, Biocenter, Innsbruck Medical University, Innsbruck, Austria

2 Department of Medical Genetics and Pharmacology, Molecular \& Clinical Pharmacology, Innsbruck Medical University, Innsbruck, Austria

* Corresponding author: A Villunger, BIOCENTER, Division of Experimental Pathophysiology \& Immunology, Innsbruck Medical University, Laboratory of Developmental Immunology, A-6020 Innsbruck, Austria.

Tel: + 43-512-507-3105; Fax: + 43-512-507-2867;

E-mail: andreas.villunger@i-med.ac.at

1. Datta SR, Brunet A, Greenberg ME. Genes Develop 1999; 13: 2905-2927.

2. Scheid MP, Woodgett JR. Nat Rev Mol Cell Biol 2001; 2: 760-768.

3. Cho $\mathrm{H}$ et al. J Biol Chem 2001; 276: 38349-38352.

4. Chen WS et al. Genes Develop 2001; 15: 2203-2208.

5. Cho H et al. Science 2001; 292: 1728-1731.

6. Easton RM et al. Mol Cell Biol 2005; 25: 1869-1878.

7. Garofalo RS et al. J Clin Invest 2003; 112: 197-208.

8. Majewski $\mathrm{N}$ et al. Mol Cell 2004; 16: 819-830.

9. Downward J. Semin Cell Dev Biol 2004; 15: 177-182.

10. Strasser A. Nat Rev Immunol 2005; 5: 189-200.

11. Dijkers PF et al. Curr Biol 2000; 10: 1201-1204.

12. Han J et al. Proc Natl Acad Sci USA 2001; 98: 11318-11323.

13. Jones RG et al. J Exp Med 2000; 191: 1721-1734.

14. Kuo ML et al. Oncogene 2001; 20: 677-685.

15. Uriarte SM et al. Cell Death Differ 2005; 12: 233-242.

16. Jones RG et al. J Immunol 2005; 175: 3790-3799.

17. Attardi LD, Donehower LA. Mutat Res 2005; 576: 4-21.

18. Zheng TS et al. Nature Med 2000; 6: 1241-1247.

19. Leonard JR et al. J Neuropathol Exp Neurol 2002; 61: 673-677.

20. Erlacher $\mathrm{M}$ et al. Blood 2005; 106: 4131-4138.

\section{DNA damage-inducing agent-elicited $\gamma$-secretase activity is dependent on $\mathrm{Bax} / \mathrm{Bcl}-2$ pathway but not on caspase cascades}

Cell Death and Differentiation (2007) 14, 189-192. doi:10.1038/sj.cdd.4402003; published online 30 June 2006

Dear Editor,

Accumulation of senile plaques composed of amyloid- $\alpha(\mathrm{A} \beta)$ is a pathological hallmark of Alzheimer's disease (AD), ${ }^{1}$ and $A \beta$ is generated through the sequential cleavage of amyloid precursor protein (APP) by $\beta$ - and $\gamma$-secretases. ${ }^{2} \beta$-Secretase excises the ectodomain of APP $(\beta \text {-APPs })^{3}$ to leave a 99amino-acid long C-terminal fragment (APP-C99-CTF) in the membrane. $\gamma$-Secretase then cleaves this membrane-tethered APP-CTF within the transmembrane domain, releasing
A $\beta$ peptides and APP-intracellular domain (AICD). As such, $\beta$ and $\gamma$-secretase are regarded to perform the key steps in the pathogenesis of $A D$ and have become important therapeutic targets in the prevention and treatment of $A D$. As a result of much effort on identifying the natures of these secretases, presenilin (PS) was found to be the first obligatory component of $\gamma$-secretase. ${ }^{4}$ Further biochemical analyses revealed that $\gamma$-secretase is composed of multicomponent complexes, and 